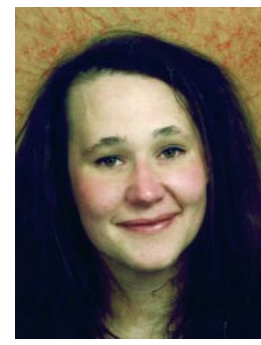

Simone Penka

\title{
Suchtprobleme bei
} Migranten - Gemeinsamkeiten und Unterschiede

\author{
Simone Penka ${ }^{1}$, Simone Krieg ${ }^{2}$, Ernestine Wohlfarth ${ }^{1}$, Andreas Heinz ${ }^{1}$ \\ ${ }^{1}$ Klinik für Psychiatrie und Psychotherapie, Campus Charité Mitte, Berlin \\ ${ }^{2}$ Zentralinstitut für Seelische Gesundheit Mannheim
}

psychoneuro 2004; 30 (7): 401-404

S uchtprobleme bei Migranten wurden lange sowohl in der Öffentlichkeit wie im Suchthilfesystem der Bundesrepublik Deutschland ignoriert. Dies spiegelt sich auch im Titel einer vor zehn Jahren in Münster 1993 durchgeführten Tagung zu Drogenfragen: „Sucht kennt keine Ausländer“. Der Tagungstitel reflektiert eindrucksvoll zwei vordergründig widersprüchliche Aspekte der Diagnostik und Behandlung von Abhängigkeitserkrankungen bei Migranten. So thematisiert er einerseits das geringe Interesse, welches das Suchthilfesystem lange Zeit Migranten als Betroffenen von Suchterkrankungen entgegenbrachte. Andererseits verweist der Titel auf die Tatsache, dass Suchtprobleme natürlich Migranten wie alle anderen in Deutschland lebenden Menschen betreffen. Sucht ist grenzenlos und macht keine Unterschiede zwischen deutschen und nichtdeutschen Suchtkranken (17).

\section{Prävalenz und Inanspruch-}

nahme des Suchthilfesystems

Obwohl bundesweit bisher keine allgemeingültigen Zahlen zu Suchterkrankungen unter Migranten erhoben wurden, liegen gerade im Bereich der Drogenabhängigkeit zahlreiche Schätzungen einzelner Städte vor. So waren in Frankfurt 1994 und 1995 ca. 37\% aller erfassten Drogenkonsumenten Nichtdeutsche (13), während in Hamburg ein Anteil von 33-35\% Abhängiger mit Migrationshintergrund festgestellt wurde (17).

Das Suchthilfesystem wird von Migranten in Deutschland nur gering in Anspruch genommen. Dies könnte eine Folge kultureller oder sozialer Zugangsbarrieren sein. Wir untersuchten die Versorgung drogenabhängiger Migranten mittels qualitativer Interviews mit Betroffenen und professionellen Helfern. Gründe für die verminderte und oft erst späte Nutzung des Suchthilfesystems waren Macht- und Perspektivlosigkeit auf dem Hintergrund von Erfahrungen der Diskriminierung und des sozialen Ausschlusses sowie Ängste um den Aufenthaltsstatus. Kulturelle Unterschiede im Verständnis von Suchterkrankungen können den Zugang zum Suchthilfesystem ebenfalls erschweren. Wir untersuchten diese Erklärungsmodelle süchtigen Verhaltens bei deutschen und türkischen Jugendlichen, d.h. einer der größten Migrantengruppen. Türkische Jugendliche lehnten viele medizinisch zentrale Begriffe zur Beschreibung und Erklärung abhängigen Verhaltens als unangemessen ab. Präventionsarbeit muss diese Unterschiede berücksichtigen, wenn sie die Migranten erreichen will.

Generell hat sich der Anteil Drogenabhängiger nichtdeutscher Herkunft in den meisten Ballungszentren in etwa dem jeweiligen Bevölkerungsanteil in den Städten angeglichen. Migranten sind offensichtlich ebenso von Suchterkrankungen betroffen wie sozial gleichgestellte Deutsche.

In den letzen Jahren ist zudem eine Zunahme der Suchtproblematik bei Menschen mit Migrationshintergrund zu erkennen (17). Wenn dies so ist, wäre gleichermaßen eine zunehmende Inanspruchnahme des Suchthilfesystems zu erwarten. Alarmierend sind deshalb Zahlen, die entgegen dieser Annahme von einer deutlich verminderten Nutzung des Suchthilfesystems durch Migranten zeugen. So machen Migranten in Frankfurter Drogenberatungsstellen lediglich $10-15 \%$ der Gesamtklientel aus (3), während sich in Hamburg der Anteil der Klienten mit Migrationshintergrund auf $8-10 \%$ beläuft (6).

Während sich also Suchtprobleme sowohl bei deutschen wie bei nichtdeutschen Bevölkerungsgruppen finden, zeigt sich ein erheblicher Unterschied in der bisher geringen Inanspruchnahme des deutschen Suchthilfesystems durch Migranten. Eine mögliche Erklärung ist das Vorhandensein von Zugangsbarrieren, die die Nutzung des Systems durch Migranten erheblich erschweren.

\section{Einflussfaktoren auf die Inanspruchnahme}

Als Gründe für die Unterrepräsentation von Migranten in der hiesigen Suchthilfe werden in der Literatur die Unkenntnis der Hilfsangebote in Deutschland, die Angst vor aufenthaltsrechtlichen Konsequenzen $^{1}$ (5), vorwiegend innerfamiliäre Bewältigungsstrategien sowie ein unzureichendes Wissen über Drogen und Abhängigkeit (14) genannt. Zudem wird auf Lösungsversuche wie das Verschicken der Erkrankten ins Heimatland (beispielsweise bei türkischen Drogenabhängigen; 18) 
sowie generell auf sprachliche und kulturelle Barrieren (3) verwiesen.

Im Rahmen der Projektgruppe „Transkulturelle Psychiatrie“ des Zentralinstituts für Seelische Gesundheit (Mannheim) und des Zentrums für Interkulturelle Psychiatrie und Psychotherapie an der Charité Berlin untersuchten wir die Zugänglichkeit von Beratungsstellen und medizinischen Versorgungseinrichtungen für Migranten mit Suchtproblemen. Die Studie bestand aus zwei Untersuchungsteilen, die zum einen die Erfahrungen der drogenabhängigen Migranten und der professionellen Helfer erhoben und zum anderen Erklärungsmodelle abhängigen Verhaltens bei deutschen und türkischen Jugendlichen erfassten. Hierzu wurden zum einen 35 Leitfadeninterviews (25 mit Drogenabhängigen nichtdeutscher Herkunft sowie 10 mit Professionellen türkischer Herkunft aus der Suchthilfe Frankfurts, Mannheims und Stuttgarts) durchgeführt (8)2. Außerdem untersuchten wir Erklärungsmodelle abhängigen Verhaltens bei über 100 deutschen und türkischen Jugendlichen (12). Zentrale Ergebnisse dieser Studien werden im Folgenden kurz dargestellt.

\section{Interviews mit Migranten und Suchthelfern}

Bei der Entwicklung und Aufrechterhaltung von Suchterkrankungen kommt neben der individuellen Disposition den sozialen Stressfaktoren, beispielsweise der Erfahrung sozialer Isolation oder sozialen Ausschlusses (2), eine wichtige Bedeutung zu (7). Bestehen kein stabiles soziales Gefüge und wenig Zukunftsperspektiven (4), sind die Chancen auf Heilung bei einer Suchterkrankung in der Regel reduziert. Vor diesem Hintergrund stellen sich die Aussagen der von uns befragten Drogenabhängigen nichtdeutscher Herkunft als sehr bedenklich dar, zeugen die Gespräche doch von einer Situation, die durch das Gefühl „nicht dazu zu gehören“, Machtlosigkeit, fehlenden Zukunftsperspektiven und Diskrimination gekennzeichnet ist. Zwar sind dies Aspekte, unter denen auch deutsche Drogenabhängige leiden, Migranten erscheinen allerdings hiervon besonders betroffen, da der Status als „Ausländer", sowohl im rechtlichen als auch im sozialen Sinn, als zusätzlicher Belastungsfaktor wahrgenommen wird und erschwerend hinzukommt. So berichtete ein Drogenabhängiger: „Mit Drogen habe ich angefangen, wo ich keine Aufenthaltsgenehmigung gekriegt habe. (...) Ich bin mit 14 in Haft gekommen und habe dann zwei Jahre abgesessen. Bin dann mit 16 raus (...). Habe mich beworben. Wollte Industriemechaniker machen. Die wollten mich auch einstellen bei einer guten Firma. Die konnten mich dann aber nicht einstellen, weil ich damals noch Zeit auf Bewährung hatte. Die wollten für den Ausbildungsvertrag mindestens drei, vier Jahre Aufenthaltsgenehmigung und damals habe ich immer nur zwei Monate gekriegt“"3.

Berichtet wurden auch Erfahrungen der Bedrohung durch Rechtsradikale, Benachteiligungen auf dem Wohnungsmarkt und deprimierende Erfahrungen auf dem Ausländeramt. Viele berichteten, ihnen sei vermittelt worden, ein „Mensch zweiter Klasse“, ein „Kanake“ oder ein „abgefuckter Türke“ zu sein. Deshalb empfänden sie sich als nicht zugehörig zur deutschen Gesellschaft. Die Belastung dieser Lebenssituation äußert sich in Reaktionen, die von Enttäuschung „Nein, Wut, Hass. Wut, Hass, und dadurch kommt auch irgendwie das ganze, dass ich auch keinen Bock habe, hier Kontakt zu haben" - bis hin zu Frustration und Resignation reichen: „Ich kämpfe nicht mehr (...). Ich kämpfe nicht, weil ich mir denke, warum soll ich kämpfen? Ich bin ein Türke irgendwie, und deswegen, warum hat der das nicht z.B., der hier geboren ist, ein Deutscher und ich muss mich mit der Ausländerbehörde in Verbindung setzen. Das kotzt mich an. Ich habe mir doch auch nicht gewünscht, (...) dass ich hier auf die Welt komme. (...).

So scheint es für die von uns Befragten weder eine stabile Lebenssituation zu geben, noch besitzen sie - auch im Rahmen der Gesetzgebung für Ausländer - eine wirkliche Perspektive in Deutschland. Denn auf eine Straftat, die auf Grund des Beschaffungsdrucks vom Drogenabhängigen begangen wird, kann der Entzug des unbefristeten Aufenthaltsstatus und somit der unsichere Verbleib in Deutschland folgen ${ }^{4}$. So erkennt beispielsweise einer der Befragten trotz zahlreicher Wunschvorstellungen (z.B. eine Lehre im Hotelfach) aufgrund seiner eingeschränkten Aufenthaltsgenehmigung keine Perspektiven für sich: „Habe ich (...) nicht [Zukunftsperspektiven]. Das ist halt auch alles kaputt jetzt mit den ganzen Straftaten und so. Und dann muss ich jetzt wieder irgendwann mal zur Ausländerbehörde“.

Unsere Untersuchung offenbarte zwei eng miteinander in Beziehung stehende Aspekte der Situation drogenabhängiger Migranten. Zum einen besteht ein deutlicher Zusammenhang zwischen der Wahrnehmung, ein „Ausländer“ zu sein und der geringen Inanspruchnahme des Suchthilfesystems. Nicht nur die wirkliche Gefahr und Angst vor aufenthaltsrechtlichen Konsequenzen (5) spielen dabei eine Rolle, sondern ebenso ein allgemeines Gefühl der

\footnotetext{
1 Bereits der Verstoß gegen das Betäubungsmittelgesetz kann für drogenabhängige Migranten erhebliche Konsequenzen nach sich ziehen. Nach §46 Abs. 4 der Ausländergesetzgebung (AuslG) kann ein Ausländer ausgewiesen werden, wenn er Heroin, Kokain oder ein vergleichbar gefährliches Betäubungsmittel verbraucht und nicht zu einer erforderlichen, seiner Rehabilitation dienenden Behandlung bereit ist. Bei Abbruch der Therapie folgt die Ausweisung bzw. Fahndung bei noch zu verbüßender Reststrafe.

2 Zur Illustration der Untersuchungsergebnisse werden Zitate aus diesen Interviews herangezogen.

3 Zwar wird bei Migranten mit gültigem oder unbefristeten Aufenthaltsstatus bei einer Erstauffälligkeit von einer Strafverfolgung abgesehen, wenn Drogenbesitz oder -handel nicht nachweisbar ist. Allerdings wies der Frankfurter Oberstaatsanwalt darauf hin, dass Migranten aufgrund ihres Ausländerstatus oftmals härter bestraft werden auf Grund eines „Missbrauches des Gastrechts“ (17), was letztendlich zur Ausweisung führen kann.

4 Wer wegen vorsätzlicher Straftaten nach dem Betäubungsmittelgesetz (BtMG) rechtskräftig zu einer Jugendstrafe von mindestens zwei Jahren oder einer Freiheitsstrafe ohne Bewährung verurteilt worden ist, kann nach §47 Abs.1, Nr.2 des AusIG ausgewiesen werden.
} 
Bedrohung und der sozialen Ausgrenzung (8). Zum anderen wird im Erleben der betroffenen Migranten ein Gefälle an Macht und Einflussmöglichkeiten deutlich, das zwischen ihnen und den Institutionen der deutschen Gesellschaft besteht. Dies ist besorgniserregend, da sich belastende Lebensereignisse, denen Migranten verstärkt ausgesetzt sind, dann besonders stark auswirken können, wenn die Betroffenen nicht das Gefühl haben, dass sie die stressvolle Situation kontrollieren oder beeinflussen können (1). Eine erhebliche Diskrepanz zwischen den reduzierten sozialen Einflussmöglichkeiten und den eigenen Wünschen kann zu Apathie und zum verstärkten Rückzug in den Drogenkonsum führen, der durch gesellschaftliche Abwertungs- und Labellingprozesse weiter stabilisiert wird (15).

\section{שrklärungsmodelle}

Das Konzept der „Erklärungsmodelle“ seelischer und körperlicher Erkrankungen wurde von Kleinman (9) entwickelt. Das kulturelle Verständnis der Entstehungsbedingungen, Symptome, Verläufe und Behandlungsmöglichkeiten einer Erkrankung bildet in seiner Gesamtheit ein Erklärungsmodell der so konstituierten Krankheit. Dieses unterliegt zwar jeweils individuellen Variationen, es finden sich jedoch wichtige Gemeinsamkeiten bei Menschen aus bestimmten gesellschaftlichen Gruppierungen und Kulturen. Zum Erklärungsmodell der Drogenabhängigkeit können auch abwertende Begriffe wie jener der „Schande“ gehören, die dazu führen, dass die Abhängigkeitserkrankung eines Familienmitglieds geheimgehalten wird (5). Unterschiedliche Erklärungsmodelle süchtigen Verhaltens zwischen Migranten und Deutschen könnten dazu beitragen, dass das deutsche Suchthilfesystem von Migranten kaum in Anspruch genommen wird.

Zur Erfassung der Erklärungsmodelle abhängigen Verhaltens

\footnotetext{
$\overline{5}$ In Deutschland treten laut Angabe des Ethnomedizinischen Zentrums Hannover bisher nur vereinzelt Ess-Störungen unter türkischen Frauen auf. Auch ihr Therapieanteil ist äußerst gering.
}

wurden dazugehörige Begriffe mit einem Fragebogen im Sinne einer freien Aufzählung bei 104 deutschen und türkischen Jugendlichen erhoben. Die 71 am häufigsten genannten Begriffe wurden einzeln auf Karten vermerkt und anschließend 20 deutschen und 20 türkischen Jugendlichen mit der Bitte vorgelegt, sie nach ihrer Zusammengehörigkeit zu sortieren. Irrelevant erscheinende Begriffe konnten aussortiert werden. Mit Hilfe einer rechnergestützten hierarchischen Clusteranalyse und einer multidimensionalen Skalierung kann dann graphisch dargestellt werden, welche Begriffe einander häufig zugeordnet wurden (12).

Interessanterweise entsprachen die begrifflichen Zuordnungen türkischer Jugendlicher eher als jene der deutschen Jugendlichen dem aktuellen Stand des medizinischen Wissens über Suchterkrankungen. So wurden Essstörungen von türkischen Jugendlichen nicht mit den stoffgebundenen Süchten, die als schwerwiegende Probleme tituliert wurden, noch mit Begriffen wie Schande oder Peinlichkeit in Verbindung gebracht. Dies war jedoch bei deutschen Jugendlichen der Fall. Türkische Jugendliche nannten die Eltern als wichtige Ansprechpartner bei Ess-Störungen, was deutsche Jugendliche nicht taten. Allerdings ordneten die türkischen Jugendlichen die Ess-Störungen den „nicht so schwerwiegenden“ Problemen zu. Dies könnte darauf hinweisen, dass Ess-Störungen wenig ernst genommen werden und dass professionelle Helfer erst spät kontaktiert werden ${ }^{5}$.

Türkische Jugendliche differenzierten im Gegensatz zu deutschen nicht zwischen den legalen Drogen (Alkohol und Nikotin) einerseits und illegalen Drogen andererseits. Erheblichen Einfluss auf diese Sichtweise könnte dabei dem Alkoholkonsumverbot in zahlreichen islamischen Glaubensrichtungen zukommen. Diese Sichtweise entspricht durchaus dem Stand des medizinischen Wissens, was schon seit Jahren auf die erhebliche Gesundheitsgefährdung der Bevölkerung durch Alkohol und Nikotin verweist, könnte aber dazu führen, dass die gesetzlichen
Konsequenzen des illegalen Drogenkonsums unterschätzt werden.

Anders als deutsche Jugendliche bezeichneten türkische Jugendliche nur die Medikamentenabhängigkeit als „psychische“ Abhängigkeit und ordneten Selbsthilfegruppen nur dieser Erkrankung als hilfreich zu. Dies deckt sich mit der in der Literatur vorzufindenden Annahme, Migranten sei oftmals das Konzept der „seelischen“ Abhängigkeit nur wenig bekannt (18) und könnte auf die Schwierigkeiten verweisen, passende Selbsthilfegruppen für Migranten $\mathrm{zu}$ finden oder $\mathrm{zu}$ initiieren.

Besonders wichtig könnten jene Begriffe sein, die von den türkischen Jugendlichen als unbrauchbar zur Beschreibung einer Suchterkrankung aussortiert wurden. Denn hier handelt es sich um zentrale Konzepte des medizinischen Abhängigkeitsbegriffs (12). So wurden u.a. jene Begriffe zurückgewiesen, die die „Kontrollminderung“ als wichtiges Kennzeichen einer Suchterkrankung bezeichnen. Die hieraus resultierende Ansicht, durchaus „ohne Suchtmittel leben“ zu können und ohne fremde Hilfe davon weg zu kommen, könnte sich als sehr gefährlich für abhängig erkrankte Migranten erweisen. Dies kam auch in den von uns geführten Interviews zum Vorschein. Hier handelt es sich sicherlich um Einstellungen, die generell bei abhängig Kranken gefunden werden können (15). Sie sind allerdings bei Migranten möglicherweise deshalb verstärkt, weil eine stärkere Eingebundenheit in die Familie und eine damit verbundene Tendenz zur Tabuisierung (5) eine größere Rolle als bei Deutschen zu spielen scheint. Begriffe von Ehre, Stolz und (vermeintlich) „männlicher“ Stärke können dazu führen, dass das institutionalisierte Hilfsangebot wenig attraktiv erscheint. So reagierten die meisten der Betroffenen auf unsere Frage, wen sie um Hilfe bitten würden, mit großer Verblüffung. „Mit Hilfe jetzt und so, ich weiß nicht, ich hab echt niemanden im Kopf daheim, draußen, versucht hat man es vielleicht schon, meine Frau, Ratschläge, aber dass ich jetzt mit meinen Problemen zu jemandem hin gehe, „Hey, was kann ich 
machen oder helf mir mal bitte“, ich weiß nicht“.

\section{Diskussion}

Suchtprobleme sind nicht nur unter der deutschen Bevölkerung anzutreffen, sondern heutzutage auch bei Migranten weit verbreitet. Sozialer Ausschluss und instabile soziale Gefüge sind wichtige Faktoren in der Entstehung und Aufrechterhaltung der Abhängigkeitserkrankungen. Migranten sind hier in besonderem Maße betroffen, da der soziale und rechtliche Ausländerstatus als zusätzlicher Belastungsfaktor hinzukommt.

Dem deutschen Suchthilfesystem gelingt es derzeit noch nicht ausreichend, nichtdeutsche Betroffene zu erreichen. Die Situation wird durch sprachliche und kulturelle Unterschiede im Umgang mit der Suchtproblematik erschwert. So zeigte sich, dass beispielsweise die Erklärungsmodelle türkischer Jugendlicher zu Suchterkrankungen in zentralen Begriffen vom medizinischen Krankheitsmodell abweichen. Will man diese Menschen erreichen, sollten die Informations- und Aufklärungsmaterialien entsprechend angepasst werden. Es scheint nicht ausreichend, für Deutsche entworfene Broschüren einfach zu übersetzen. Auch im therapeutischen Gespräch können diese sprachlich-konzeptionellen Unterschiede die Verständigung erschweren und sollten soweit wie möglich beachtet werden.

So wichtig und erfolgversprechend im einzelnen Anstrengungen sind, die Inanspruchnahme psychosozialer Beratungsstellen durch optimierte und vermehrte Information der Migranten zu verbessern, so wenig können derartige Anstrengungen allein das Gefühl von Hilf- und Machtlosigkeit beseitigen, das viele drogenabhängige Migranten beeinträchtigt. Auch ausländerpolitische Maßnahmen und die oft als fremdenfeindlich erfahrene Stimmung in Deutschland beeinflussen die Inanspruchnahme des Suchthilfesystems. Eine wichtige Voraussetzung für den angemessenen Umgang mit drogenabhängigen Migranten ist auch die Bereitschaft der deutschen Gesellschaft, die Realität der Einwanderung anzuerkennen und Mig- ranten wie Abhängigkranke mit deutschem Pass zu behandeln. Der Grundsatz „Therapie statt Strafe“ sollte auch bei Migranten den Umgang mit abhängig Kranken leiten.

\section{In Germany, immigrants rarely use} the medical system for drug addiction. Access to medical treatment may be restricted by cultural or social barriers. Qualitative interviews were used to examine treatment options for and experience of drug addicted migrants and professionals. Migrants reported feelings of helplessness, lack of influence, discrimination, social exclusion, and fears to lose the right to stay in Germany as explanations for reduced or late utilization of the medical system. Cultural differences in the understanding and interpretation of drug addiction may also contribute to reduced access to professional treatment. Explanatory models of addictive behavior were examined in German and Turkish youth, i.e. one of the largest immigrant groups in Germany. Turkish youth rejected many important medical expressions as inadequate to describe addiction. Such differences in explanatory models should be considered if preventive efforts are supposed to reach immigrant populations.

\section{Literatur}

1. Antonovsky A. Meine Odyssee als Stressforscher. In: Jahrbuch für Kritische Medizin 1991; Argument-Sonderband AS 193, 17: $112-130$

2. Blakey M. Psychophysiological stress and disorders of industrial society: a critical theoretical formulation for biocultural research. In: Forman S (Hrsg.). Diagnosing America. Anthropology and public engagement. Ann Arbor, University of Michigan Press, 1994: 149-192

3. Gaitanides S. Zugangsbarrieren von Migranten zu den Drogendiensten. In: Deutsche Hauptstelle gegen die Suchtgefahren (Hrsg.). Sucht in unserer multikulturellen Gesellschaft. Freiburg i.Br., Lambertus-Verlag, 1998, 62-76

4. Gastpar M et al. (Hrsg.). Lehrbuch der Suchterkrankungen. Stuttgart, Thieme, 2000: 50-69

5. Grüsser $S$ et al. Drogenabhängigkeit und Migration innerhalb der Europäischen Union (EU). In: Gölz: Moderne Suchtmedizin 1999. 5/B7: 1-5

6. Haasen $\mathrm{C}$ et al. Psychosoziale Aspekte der Sucht bei Migranten. Suchttherapie. Prävention, Behandlung, wissenschaftliche Grundlagen 2001; 2: 161-166

7. Heinz A. Serotonerge Dysfunktion als
Folge sozialer Isolation - Bedeutung für die Entstehung von Aggression und Alkoholabhängigkeit. Nervenarzt 1999; 70: 780-789

8. Hunner $C$ et al. Latente Ausschliessung: Migranten und Drogenhilfe. Kriminologisches Journal 2001; 3: 216-224

9. Kleinman A. Patients and healers in the context of culture. An Exploration of the borderland between Anthropology, Medicine, and Psychiatry. Berkeley, Los Angeles und London, University of California Press, 1980

10. Körner HH. Juristische Aspekte der Drogenkriminalität und Möglichkeiten der Hilfeleistung für Drogenabhängige. In: Kommunale Ausländerinnen- und Ausländervertretung (KAV) der Stadt Frankfurt am Main (Hrsg.). Drogen und Migration: Dokumentation der Anhörung am 4. März 1996 zur Situation drogenabhängiger und gefährdeter junger Menschen ausländischer Herkunft in Frankfurt am Main. Bonn, Forum-Verlag Godesberg, 1996: 33-49

11. Krieg $S$ et al. Körperbilder und Essstörungen türkischer Migrantinnen. Psychomed. Zeitschrift für Psychologie und Medizin 2002; 2: 80-84

12. Penka $\mathrm{S}$ et al. Unterschiedliche Erklärungsmodelle für abhängiges Verhalten bei türkischen und deutschen Jugendlichen - Bedeutung für Prävention und Behandlungsangebote. Nervenarzt (im Druck)

13. Philippi R. Drogenkriminalität und Gefährdungspotential. In: Kommunale Ausländerinnen- und Ausländervertretung (KAV) der Stadt Frankfurt am Main (Hrsg.). Drogen und Migration. Dokumentation der Anhörung am 4. März 1996 zur Situation drogenabhängiger und -gefährdeter junger Menschen ausländischer Herkunft in Frankfurt am Main. Bonn, Forum-Verlag Godesberg, 1996: 67-72

14. Salman R. Stand und Perspektiven interkultureller Suchthilfe. In: Salman R et al. (Hrsg.). Handbuch Interkultureller Suchthilfe. Modelle, Konzepte und Ansätze der Prävention, Beratung und Therapie. Giessen, Psychosozial-Verlag, 1999: 11-30 15. Schmidt B. et al. Soziale Bedingungsfaktoren von Drogenkonsum und Drogenmissbrauch. In: Gastpar M et al. (Hrsg.). Lehrbuch der Suchterkrankungen. Stuttgart, Thieme, 2000: 50-69

16. Scholl M. Begrüssungsrede. In: Landschaftsverband Westfalen-Lippe (Hrsg.). 13. Jahrestagung: Sucht kennt keine Ausländer, Dokumentation. Münster, 1994: 1-5

17. Toprak MA et al. Sucht. In: Haasen C et al. (Hrsg.). Beurteilung psychischer Störungen in einer multikulturellen Gesellschaft. Freiburg i.Br., Lambertus-Verlag, 2000: $145-164$

18. Umminger $G$. Keiner liebt sie, keiner versteht sie. Suchtreport 1997; 6: 6-13

\section{Korrespondenzadresse:}

Prof. Dr. A. Heinz

Klinik für Psychiatrie und Psychotherapie der Charité

Humboldt-Universität zu Berlin

Schumannstr. 20/21

10117 Berlin

E-Mail: andreas.heinz@charite.de 